\title{
Prevalence and Antibiotic Resistance Pattern of Acinetobacter Isolated from Patients Admitted in ICUs in Mazandaran, Northern Iran
}

\author{
Roya Ghasemian ${ }^{1}$, Mohammad Ahanjan ${ }^{2}$, Ebrahim Fatehi $^{3} \&$ Mehran Shokri $^{4}$ \\ ${ }^{1}$ Department of Infectious diseases, Antimicrobial Resistance Research Center, Mazandaran University of \\ Medical Sciences, Sari, Iran \\ ${ }^{2}$ Department of Microbiology, Antimicrobial Resistance Research Center Mazandaran University of Medical \\ Sciences, Sari, Iran \\ ${ }^{3}$ Department of Infectious diseases, Antimicrobial Resistance Research Center, Mazandaran University of \\ Medical Sciences, Sari, Iran \\ ${ }^{4}$ Infectious Diseases and Tropical Medicine Research Center, Babol University of Medical Sciences, Babol, Iran \\ Correspondence: Mehran Shokri, Infectious Diseases and Tropical Medicine Research Center, Babol University \\ of Medical Sciences, Babol, Iran. Tel: 98-912-293-7701. E-mail: mshokri2015@yahoo.com
}

Received: November 2, 2015 Accepted: January 27, 2016 Online Published: February 29, 2015

doi:10.5539/gjhs.v8n11p112 URL: http://dx.doi.org/10.5539/gjhs.v8n11p112

\begin{abstract}
Background and Purpose: Antibiotic resistance rate is increasing in Acinetobacter species, especially in Acinetobacter baumannii, as the most important pathogen of hospital and ICU . This research aimed to evaluate antibiotic resistant rate of Acinetobacter spp. isolated from patients admitted to ICUs in educational hospitals affiliated with Mazandaran University of Medical Sciences.
\end{abstract}

Methods: In this cross-sectional descriptive study, 50 Acinetobacter isolates were collected during 2013- 2014. After confirming Acinetobacter species, antibacterial sensitivity test was done using disc diffusion method and minimal inhibitor concentration (MIC) was evaluated by E-test in all isolates.

Results: Disc diffusion method revealed that $100 \%$ of isolates were resistant to Amikacin and Cefepim and $96 \%$ were resistant to both Meropenem and Ciprofloxacin antibiotics, $6 \%$ were sensitive, $18 \%$ were intermediate and $76 \%$ were resistant to imipenem. Also, $84 \%$ of isolates were sensitive and $16 \%$ were resistant to colistin. In E-test method, $92 \%$ of isolates were sensitive and $8 \%$ were resistant to colistin. Moreover, an isolate was sensitive, one was intermediate and the remaining isolates were resistant to ciprofloxacin, and $100 \%$ of isolates were resistant to other antibiotics in E-test. Over $96 \%$ of Acinetobacter isolates were resistant to the antibiotics frequently used in ICU (ciprofloxacin, meropenem, amikacin, and cefepim). Colistin was found as the only appropriate antibiotic that could be used for patients in ICU.

Conclusion: We hope these results could change the attitude of physicians toward using antibiotics in ICUs and encourage them to follow antibiotic stewardship as the only effective strategy to somewhat control antibiotic resistances.

Keywords: antibiotic resistance, Acinetobacter, disc diffusion, E-test, ICU

\section{Introduction}

Acinetobacter is a group of ever-evolving opportunist pathogens which affects various groups of people, especially patients hospitalized in ICU (Ramphal \& Ambrose, 2006). This is a non-fermentative, nonmotile, gram-negative bacteria commonly found in water and soil. Acinetobacter lives as normal flora in healthy people's Oropharynx and in recent years have been reported as a major factor in hospital infection (Hanlon, 2005; $\mathrm{Ku}$ et al., 2000). The most important species of this organism is Acinetobacter baumannii which causes different types of infections including respiratory system infection, urinary tract infection, blood and wound infection, particularly in ICU. More over this organism forms biofilms on abiotic surfaces, a phenotype that can enucleate its ability to survive in nosocomial environments and to cause infections with devices in immunocompromised cases (He et al., 2015). In recent years, different species of Acinetobacter have become even more resistant (Coelho et al., 2004). 
Acinetobacter has an acquired resistance to most available antimicrobial agents including Aminoglycosides, Quinolones and Extended-spectrum beta-lactamases. High rate of antimicrobial resistance of these bacteria, along with their endurance and survival ability, has made them a serious threat to hospital environments in developed countries and other places. Most of the species are resistant to Cephalosporins and resistance to Carbapenems is exactly growing too (Shakibaie et al., 2011).

Acinetobacter baumannii is the most widespread opportunistic pathogen responsible for hospital infection that frequently causes severe infections in high-risk populations including old people, premature children, newborns, operated patients, individuals undergoing peritoneal dialysis, patients with tracheostomy tubes, severely burned patients, those with tracheal intubation, mechanical ventilation, intravenous catheters and people who are treated with extended-spectrum antibiotics or Immunosuppressives (Zheng et al., 2013).

Based on the increasing reports of Acinetobacter species isolated from patients, especially in ICU, and growth of Acinetobacter strains' resistance to new and available antibiotics and high cost of using antibiotics, recognizing the resistance patterns is necessary in every center. On the other hand, isolating Acinetobacter species from clinical patients does not necessarily prove the presence of infection and should be evaluated according to the clinical conditions of the patients. In this situation, choosing the right method of treatment requires knowing the periodic pattern of the resistance. Therefore, in addition to studying the clinical importance of isolated species of Acinetobacter and determining the risk factors associated with clinical infection, in this study we aimed at investigating the resistance rate of isolated species in patients in four ICUs of educational hospitals of Mazandaran University of Medical Sciences.

\section{Materials and Methods}

In this study, 50 samples of Acinetobacter bacteria were isolated from ICUs in educational hospitals affiliated to Mazandaran University of Medical Sciences (Razi, Imam Khomeini, Fatemeh zahra and $\mathrm{Bu}$ ali sina). After transferring the clinical samples to the lab (department of microbiology, SARI Medical school), they were cultured in Bacteriological blood agar and eosin methylene blue (EMB) environments. Then, plates were incubated in aerobic condition at 37 degrees centigrade for 24 hours. Subsequently, culture environments were studied and if any growth was seen subtractive tests were done immediately. In this way, gram stain was done and gram-negative bacilli and oxidase were identified. Then using biochemical tests including Citrate test, Urea test, Indole test, movement test and oxidative-fermentation test and growth in 42-44 degrees centigrade, the presence of Acinetobacter was proved. The sensitivity of antibacterial was then determined using Disk Diffusion method based on CLSI standard on Mueller Hinton-agar environment. For this purpose microbial suspension in accordance with the density of half McFarland was prepared and spread on the aforementioned environment. For the isolated Acinetobacter, antibiogram test was conducted using antibiotic disks with Colsitin $(110 \mu \mathrm{g})$, Cefepime $(30 \mu \mathrm{g})$, Amikacin $(30 \mu \mathrm{g})$, Imipenem $(30 \mu \mathrm{g})$, Meropenem $(30 \mu \mathrm{g})$, and Ciprofloxacin $(30 \mu \mathrm{g})$. E-test method was used to determine MIC in isolations that exhibited high resistance in Disk Diffusion method. E-test strip films were provided from HIMEDIA, India and Liofilchem, Italy. All patients were followed for 3 to 6 weeks, and the outcome including death or recovery was studied. If patients were discharged their condition was followed via phone contacts.

\subsection{Statistical Analysis}

The information was collected and qualitative data was analyzed in SPSS (Ver. 17) and quantitative data was analyzed applying ANOVA.

\section{Results}

During 2013- 2014, we studied 50 samples of Acinetobacter bacteria isolated from 50 patients (58\% men) among ICUs in Razi $(n=25)$, Imam Khomeini $(n=17)$, Fatemeh zahra $(n=3)$ and Bu ali sina $(n=5)$ hospitals in Mazandaran province. Most of the patients were aged60-70 (38\%) and 70-80 (36\%) years. Also, 6 patients (12\%) were over 80,4 patients $(8 \%)$ were $50-60$ and 3 patients $(6 \%)$ were under 50 years of age. The mean period of hospitalization was $12.72 \pm 4.45$ days. No significant difference was found between the hospitals regarding the period of hospitalization in ICU $(\mathrm{p}=0.74)$. The samples were isolated 5 to 12 days after hospitalization. Before culturing Acinetobacter, most of the patients received different types of antibiotics and more than $80 \%$ received two antibiotics. The most common antibiotics used for these patients were Imipenem, Meropenem, Vancomycin, Tazocin, Ciprofloxacin, Ceftriaxone, and Clindamycin.

The most prevalent causes of illness were sepsis (22\%) pneumonia (20\%), multiple trauma and soft tissue infection (12\% each), hematologic disorders and neurosurgery (10\% each), cerebral stroke $(6 \%)$, abdominal surgery and poisoning $(4 \%$ each). The most common place of sample isolation was endo-tracheal tube (ETT) $(n=$ 
$35,70 \%)$. Other places of sample isolation included wound sites $(n=5,10 \%)$, blood culture $(B / C)$ and urinary culture $(\mathrm{U} / \mathrm{C})(\mathrm{n}=3$ each, $6 \%)$ and cerebrospinal fluid culture $(\mathrm{CSF} / \mathrm{C})$, Darren, Bronchoalveolar lavage (BAL) and Tracheostomy ( $\mathrm{n}=1$ each, 2\%). Antibiogram results using disc diffusion and E-test method are presented in table 1 and 2, respectively. The patients were followed for 3 to 6 weeks. Death occurred in 31 cases $(62 \%)$ and 19 patients $(38 \%)$ recovered.

Table 1. Results of the antibiogram of cultivations using disk diffusion (for Acinetobacter)

\begin{tabular}{lllll}
\hline Antibiotic & Sensitive & Intermediate & Resistant & Total \\
\hline amikacin & 0 & 0 & $50(100 \%)$ & $50(100 \%)$ \\
imipenem & $3(6 \%)$ & $9(18 \%)$ & $38(76 \%)$ & $50(100 \%)$ \\
ciprofloxacin & $2(4 \%)$ & 0 & $48(96 \%)$ & $50(100 \%)$ \\
colistin & $42(84 \%)$ & 0 & $8(16 \%)$ & $50(100 \%)$ \\
meropenem & $2(4 \%)$ & 0 & $48(96 \%)$ & $50(100 \%)$ \\
cefepime & 0 & 0 & $50(100 \%)$ & $50(100 \%)$ \\
\hline
\end{tabular}

Table 2. Results of the antibiogram of cultivations using E-Test (for Acinetobacter)

\begin{tabular}{lllll}
\hline Antibiotic & Sensitive & Intermediate & Resistant & Total \\
\hline amikacin & 0 & 0 & $50(100 \%)$ & $50(100 \%)$ \\
colistin & $46(92 \%)$ & 0 & $4(8 \%)$ & $50(100 \%)$ \\
ciprofloxacin & $1(2 \%)$ & $1(2 \%)$ & $48(96 \%)$ & $50(100 \%)$ \\
imipenem & 0 & 0 & $50(100 \%)$ & $50(100 \%)$ \\
meropenem & 0 & 0 & $50(100 \%)$ & $50(100 \%)$ \\
cefepime & 0 & 0 & $50(100 \%)$ & $50(100 \%)$ \\
\hline
\end{tabular}

Table 3. Comparison of resistant average (\%) in different studies in Iran and current research

\begin{tabular}{lllllll}
\hline $\begin{array}{l}\text { Antibiotic } \\
\text { study }\end{array}$ & amikacin & imipenem & ciprofloxacin & colistin & meropenem & cefepime \\
\hline $2001-2007$ & 58.4 & 51.1 & 83.9 & - & 64.3 & - \\
$2007-2008$ & 79.4 & 32 & 67.6 & 1.3 & 27.8 & 94.1 \\
$2008-2009$ & 82.7 & 57.75 & 83.75 & 19 & 59.25 & 89 \\
$2009-2010$ & 69.5 & 52.4 & 92 & 12 & 72 & 96.6 \\
$2010-2011$ & 75 & 81.9 & 85.2 & 9.3 & 85.2 & 88.6 \\
$2011-2012$ & 89.5 & 80.5 & 97 & - & 81.5 & - \\
$2012-2014$ & 95 & 76.5 & 72 & 16 & 81.5 & 97 \\
disk diffusion & 100 & 76 & 96 & 16 & 96 & 100 \\
E-test & 100 & 100 & 96 & 8 & 100 & 100 \\
\hline
\end{tabular}

\section{Discussion}

This study aimed to evaluate the prevalence of antibiotical resistance of Acinetobacter species isolated from 50 patients hospitalized in ICUs of four University affiliated hospitals. Environmental flexibility and extended-spectrum of resistive variable, has made Acinetobactera very dangerous nosocomial pathogen (Nordmann, 2004). There are many accounts of Multi-drug resistance (MDR) Acinetobacter baumannii in the hospitals of Europe, North America, Argentina, Brazil, China, Taiwan, Hong Kong, Japan, Korea and even far regions such as Haiti and South Pole (Barbolla et al., 2003; Houang et al., 2001; Levin et al., 1996; Liu et al., 2006; Naas et al., 2005; Nishio et al., 2004). The diffusion of MDR strains often causes these epidemics at the 
level of cities, countries and continents (Barbolla et al., 2003; Da Silva et al., 2004; Landman et al., 2002). It is proved that MDR strains are transferred from regions with high antimicrobial resistance rate to regions with lower rates from Spain to Norway (Onarheim et al., 2000).

Our results revealed a very high rate of resistance to Cefepim (100\%).

In order to evaluate such high rate of resistance, we should consider the mechanisms of drug resistance of Acinetobacter baumannii to Cephalosporins. Although it has been clear that TEM-1 beta-Lactamases occurs in Acinetobacter baumannii, recently, Extended-Spectrum Beta-Lactamases (ESBL) was also noted in Acinetobacter baumannii (Vila et al., 1993). Strains of Acinetobacter baumannii which carry PER-1 (1 ESBL) show high resistance to Extended-Spectrum Cephalosporins and Penicillins, however fortunately they do not interfere with the resistance of Acinetobacter baumannii to Carbapenems (Perez et al., 2007). All the samples were resistant to Amikacin which was confirmed by E-test method.Acinetobacter baumannii resistance to Aminoglycosides is mainly attributed to EffluxED ABC pumps and Aminoglycoside changer enzymes. These enzymes include Aminoglycoside Phosphotransferases, Aminoglycoside transferases style, and Aminoglycoside nucleotidyl transferase (Nemec et al., 2004; Vila et al., 1997).

Current study showed high rate of resistance to Ciprofloxacin too. Actually only 2 samples (4\%) showed sensitivity to Ciprofloxacin. In E-test, one sample was identified to be sensitive (50\%) and the other one had intermediate sesitivity (50\%) to Ciprofloxacin. Resistance of Acinetobacter baumanniito Quinolones is often due to changes in DNA structure of gyrA, which is secondary to mutations in areas that determine resistance to quinolones in gyrA and parC genes (Seward \& Towner, 1998; Vila, et al., 1997). These changes reduce tendency to connect quinolones to DNA-enzyme complex. Another mechanism of resistance to quinolones in created by efflux systems which decreases the aggregation of drug in intercellular spaces (Heinemann et al., 2000).

Few studies have reported the resistance of Acinetobacte rbaumannii to Colistin, which is a strong warning (Gales et al., 2001; Urban et al., 2001). Urban et al. (2001) have found a case of Acinetobacter baumannii resistance to Polymyxin B (Urban, et al., 2001). Mechanism of resistance to Colistin is probably related to changes in Acinetobacter baumannii Lipopolysaccharide (e. g. Acidification, Acylation or the presence of intermediary antigens in connecting antibiotic to cell membrane) (Peterson et al., 1987). Although in our study the highest rate of Acinetobacter baumannii sensitivity was to Colistin, but out of the 50 samples studied using Disk Diffusion, 8 samples (16\%) were resistant to Colistin. However by E-test the resistance pattern was only confirmed in half of them (4 species, $8 \%$ )

Resistance rates of Acinetobacter to Carbapenems were as follows: $96 \%$ of the samples were resistant to Meropenem and $76 \%$ were resistant to Imipenem. Also, 9 isolates had intermediate sensitivity to Imipenem (18\%). Acinetobacter baumannii resistance to Carbapenems was significantly related to Beta-lactamases of group B and D. There is Metallo-beta-lactamase in group B, which hydrolyzes Carbapenems and other Beta-lactam antibiotics except in Aztreonam (Walsh, 2005). On the other hand, in Beta-Lactamases of group D, resistance is secondry to the exsistace of the OXA, which is very alarmingdue to deactivation of Carbapenems. The first description of an OXA Carbapenemase in Acinetobacter baumannii was OXA-23 which was isolated from a clinical sample in Scotland before Carbapenem was discovered (Marqué et al., 2005).

In the study of Juyal et al. (2013) in a tertiary level hospital in India, the sensitivity rate of Acinetobacter was $73.61 \%$ to Amikacin, $68.06 \%$ to Imipenem, $36.11 \%$ to Ticarcillin/ Clavulanic acid, $29.17 \%$ to Piperacillin/ Tazobactam, $48.61 \%$ to Gentamicin, $31.94 \%$ to Cefoperazone/Sulbactam, $26.39 \%$ to Cefepime, $16.67 \%$ to Piperacillin, $18.06 \%$ to Cefoperazone and Ceftazidime, $12.5 \%$ to Ciprofloxacin, $8.33 \%$ to Aztreonam, and $23.61 \%$ to Cotrimoxazole. The sensitivity rate in this study is generally higher than what has been seen in current study, especially in the case of Amikacin and Imipenem. Strategies of antibiotic consumption and the time of study definitely have a major role in these differences.

Inanotherstudy (Shakibaie, et al., 2011) performed on ICUs in Iran, resistance to Imipenem was $73.3 \%$ and resistance to Cefepime was $93.3 \%$, which are similar to our findings, while the resistance rates to Ciprofloxacin and Amikacin were 66\% and 53.3\%, respectively which were much lower than the rate observed in our study.

In a systematic review, antibiotic resistance rate of Acinetobacter baumannii in samples of Iranian patients was evaluated (Moradi et al., 2015) among 3409 samples collecting during 2001 to 2013.The study showed a significant increase in resistance rate against Imipenem and Meropenem, while resistance to lipopeptides and Aminoglycosides did not have a considerable change during these years. Resistance to Carbapenems at the beginning of the research (2001) was low (51.1\% for Imipenem and $64.3 \%$ for Meropenem) but at the end of the study (2013) it reached $76.5 \%$ for Imipenem and $81.5 \%$ for Meropenem, showing similar resistance rates to our results. That study also showed that prevalence of MDR Acinetobacter baumannii significantly increased during 
this period. In this study, resistance to Colistin was between 1.3\% to $19 \%$ (the latter was similar to our findings). Comparison of this review to the present study are shown in Table 3.

Nowadays, effective antibiotics for cure of Acinetobacter baumannii infections include: Aminoglycosides, Fluoroquinolones and Carbapenems (Falagas, et al., 2005) however, because of the high rate of Acinetobacter baumannii resistance to these antibiotics, they cannot be used as empirical treatment. For example, Carbapenems which are commonly prescribed for patients infected with life-threatening Acinetobacter baumannii, have the highest rate of resistance compared to other antibiotics. These facts could explain the reason of therapeutic failures following life-threatening infections with strains resistant to Carbapenem. Recently, Colistin and Tigecycline have emerged as alternative treatment choice for MDR Acintobacter infections. However, resistance to these antimicrobial agents has also been reported as a result of the increased usage of colistin and tigecycline (Capon et al., 2008).

Although resistance to Colistin is still low, but our study showed increasing resistance to this group which could be problematic in the future. The emergence of resistance has been experienced rapidly after its widespread use (Li et al., 2006; Gounden et al., 2009; Park et al., 2009).

Thus, many recent studies have investigated combined therapy of two or more agents for MDR acintobacter infections. Specially combination regimen of Tigecycline or Colistin with other antibiotics have frequently been reported .Park and coworker found a high rate of in vitro synergy and bactericidal activity and lack of antagonism in combination of colistin and Doripenem (Park et al., 2016). As well as, recent study revealed a superior activity for combination of tigecycline with cefoperazone-sulbactam against MDR acintobacter (Liu et al., 2014).

\section{Conclusion}

Our results clearly showed a very great rate of resistance against most of the antibiotics, usually prescribed for aforementioned infections. There were more than $96 \%$ rates of resistance against the antibiotics frequently used in ICU wards (Cefepime, Amikacin, Ciprofloxacin, Imipenemand Meropenem). These results prove that drug resistance, especially against Carbapenem, is increasing fast. Monotherapy should be avoided,because of rapid emerging of resistant, even with Colistin or Tigecycline. Recently combination regimen of the Colistin or Tigecycline with other antibiotics like Cefoprazon-sulbactam or Doripenem are promising in treatment of severe infection specially by MDR acintobacter.

We also hope these results could change the attitude of physicians toward using antibiotics in ICUs and encourage them to follow antibiotic stewardship as the only effective strategy to somewhat control antibiotic resistance in healthcare settings.

\section{Acknowledgments}

The authors thank Deputy of Research and Technology of Mazandaran University of Medical Sciences.

\section{Funding}

The study was a thesis of residency and received a grant from Mazandaran University of Medical Sciences. (Grant number: 454).

\section{Conflict of Interest}

The authors declare that there is no conflict of interests regarding the publication of this paper.

\section{References}

Barbolla, R. E., Centrón, D., Di Martino, A., Maimone, S., Salgueira, C., Famiglietti, A., . . Catalano, M. (2003). Identification of an epidemic carbapenem-resistant Acinetobacter baumannii strain at hospitals in Buenos Aires City. Diagnostic microbiology and infectious disease, 45(4), 261-264. http://dx.doi.org/10.1016/S0732-8893(02)00538-2

Capone, A., D'Arezzo, S., Visca, P., \& Petrosillo, N. (2008). In vitro activity of tigecycline against multidrug-resistant Acinetobacter baumannii. Journal of antimicrobial chemotherapy, 62(2), 422-423. http://dx.doi.org/10.1093/jac/dkn172

Coelho, J., Woodford, N., Turton, J., \& Livermore, D. (2004). Multiresistant acinetobacter in the UK: how big a threat? Journal of hospital infection, 58(3), 167-169. http://dx.doi.org/10.1016/j.jhin.2003.12.019

Da Silva, G., Quinteira, S., Bertolo, E., Sousa, J., Gallego, L., Duarte, A., . . Group, P. R. S. (2004). Long-term dissemination of an OXA-40 carbapenemase-producing Acinetobacter baumannii clone in the Iberian Peninsula. Journal of Antimicrobial Chemotherapy, 54(1), 255-258. http://dx.doi.org/10.1093/jac/dkh269 
Falagas, M. E., Kasiakou, S. K., \& Saravolatz, L. D. (2005). Colistin: the revival of polymyxins for the management of multidrug-resistant gram-negative bacterial infections. Clinical infectious diseases, 40(9), 1333-1341. http://dx.doi.org/10.1086/429323

Gales, A. C., Reis, A. O., \& Jones, R. N. (2001). Contemporary assessment of antimicrobial susceptibility testing methods for polymyxin B and colistin: Review of available interpretative criteria and quality control $\begin{array}{lllll}\text { guidelines. Journal of clinical microbiology, } & 39(1), & 183-190 .\end{array}$ http://dx.doi.org/10.1128/JCM.39.1.183-190.2001

Gounden, R., Bamford, C., van Zyl-Smit, R., Cohen, K., \& Maartens, G. (2009). Safety and effectiveness of colistin compared with tobramycin for multi-drug resistant Acinetobacter baumannii infections. BMC infectious diseases, 9(1), 26. http://dx.doi.org/10.1186/1471-2334-9-26

Han, X., Li, Q., Shen, L., Hu, D., \& Qu, Y. (2014). [Correlation between the biofilm-forming ability, biofilm-related genes and antimicrobial resistance of Acinetobacter baumannii]. Zhonghua Wei Zhong Bing Ji Jiи Yi Xие, 26(9), 639-43. doi:10.3760/cma.j.issn.2095 4352.2014.09.007.(abstract)

Hanlon, G. (2005). The emergence of multidrug resistant Acinetobacter species: a major concern in the hospital $\begin{array}{lllll}\text { setting. Letters in } & \text { microbiology, }\end{array}$ http://dx.doi.org/10.1111/j.1472-765X.2005.01791.x

He, X., Lu, F., Yuan, F., Jiang, D., Zhao, P., Zhu, J., Cheng, H., Cao, J., \& Lu, G. (2015). Biofilm formation caused by clinical Acinetobacter baumannii isolates is associated with overexpression of the AdeFGH efflux pump. Antimicrob Agents Chemother, 59, 4817-4825. http://dx.doi.org/10.1128/AAC.00877-15

Heinemann, B., Wisplinghoff, H., Edmond, M., \& Seifert, H. (2000). Comparative activities of ciprofloxacin, clinafloxacin, gatifloxacin, gemifloxacin, levofloxacin, moxifloxacin, and trovafloxacin against epidemiologically defined Acinetobacter baumannii strains. Antimicrobial agents and chemotherapy, 44(8), 2211-2213. http://dx.doi.org/10.1128/AAC.44.8.2211-2213.2000

Houang, E. T., Chu, Y., Leung, C., Chu, K., Berlau, J., Ng, K., \& Cheng, A. (2001). Epidemiology and Infection Control Implications ofAcinetobacter spp. in Hong Kong. Journal of clinical microbiology, 39(1), 228-234. http://dx.doi.org/10.1128/JCM.39.1.228-234.2001

Juyal, D., Prakash, R., Shanakarnarayan, S. A., Sharma, M., Negi, V., \& Sharma, N. (2013). Prevalence of non-fermenting gram negative bacilli and their in vitro susceptibility pattern in a tertiary care hospital of Uttarakhand: A study from foothills of Himalayas. Prevalence, 2(2), 108-112. http://dx.doi.org/10.4103/2278-0521.117915

Ku, S., Hsueh, P., Yang, P., \& Luh, K. (2000). Clinical and microbiological characteristics of bacteremia caused by Acinetobacter lwoffii. European Journal of Clinical Microbiology and Infectious Diseases, 19(7), 501-505. http://dx.doi.org/10.1007/s100960000315

Landman, D., Quale, J. M., Mayorga, D., Adedeji, A., Vangala, K., Ravishankar, J., . . Brooks, S. (2002). Citywide clonal outbreak of multiresistant Acinetobacter baumannii and Pseudomonas aeruginosa in Brooklyn, NY: the preantibiotic era has returned. Archives of internal medicine, 162(13), 1515-1520. http://dx.doi.org/10.1001/archinte.162.13.1515

Levin, A. S., Mendes, C. M., Sinto, S. I., Sader, H. S., Scarpitta, C. R., Rodrigues, E., . . Boulos, M. (1996). An outbreak of multiresistant Acinetobacter baumanii in a university hospital in Sao Paulo, Brazil. Infection Control, 17(6), 366-368. http://dx.doi.org/10.1086/647319

Liu, B., Bai, Y., Liu, Y., Di, X., Zhang, X., Wang, R., \& Wang, J. (2014). In vitro activity of tigecycline in combination with cefoperazone-sulbactam against multidrug-resistant Acinetobacter baumannii. Journal of Chemotherapy, 1973947814 Y-0000000203.

Liu, S., Lin, J., Chu, C., Su, L., Lin, T., \& Chiu, C. (2006). Integron-associated imipenem resistance in Acinetobacter baumannii isolated from a regional hospital in Taiwan. International journal of antimicrobial agents, 27(1), 81-84. http://dx.doi.org/10.1016/j.ijantimicag.2005.09.010

Li, J., Rayner, C. R., Nation, R. L., Owen, R. J., Spelman, D., Tan, K. E., \& Liolios, L. (2006). Heteroresistance to colistin in multidrug-resistant Acinetobacter baumannii. Antimicrobial agents and chemotherapy, 50(9), 2946-2950. http://dx.doi.org/10.1128/AAC.00103-06

Marqué, S., Poirel, L., Héritier, C., Brisse, S., Blasco, M. D., Filip, R., . . Nordmann, P. (2005). Regional occurrence of plasmid-mediated carbapenem-hydrolyzing oxacillinase OXA-58 in Acinetobacter spp. in 
Europe. Journal of clinical microbiology, 43(9), 4885-4888. http://dx.doi.org/10.1128/JCM.43.9.4885-4888.2005

Moradi, J., Hashemi, F. B., \& Bahador, A. (2015). Antibiotic Resistance of Acinetobacter baumannii in Iran: A Systemic Review of the Published Literature. Osong public health and research perspectives, 6(2), 79-86. http://dx.doi.org/10.1016/j.phrp.2014.12.006

Naas, T., Levy, M., Hirschauer, C., Marchandin, H., \& Nordmann, P. (2005). Outbreak of carbapenem-resistant Acinetobacter baumannii producing the carbapenemase OXA-23 in a tertiary care hospital of Papeete, French Polynesia. Journal of clinical microbiology, 43(9), 4826-4829. http://dx.doi.org/10.1128/JCM.43.9.4826-4829.2005

Nemec, A., Dolzani, L., Brisse, S., van den Broek, P., \& Dijkshoorn, L. (2004). Diversity of aminoglycoside-resistance genes and their association with class 1 integrons among strains of pan-European Acinetobacter baumannii clones. Journal of medical microbiology, 53(12), 1233-1240. http://dx.doi.org/10.1099/jmm.0.45716-0

Nishio, H., Komatsu, M., Shibata, N., Shimakawa, K., Sueyoshi, N., Ura, T., . . . Wada, Y. (2004). Metallo- $\beta$-lactamase-producing gram-negative bacilli: laboratory-based surveillance in cooperation with 13 clinical laboratories in the Kinki region of Japan. Journal of clinical microbiology, 42(11), 5256-5263. http://dx.doi.org/10.1128/JCM.42.11.5256-5263.2004

Nordmann, P. (2004). Acinetobacter baumannii, le pathogène nosocomial par excellence. Pathologie Biologie, 52(6), 301-303. http://dx.doi.org/10.1016/j.patbio.2004.03.007

Onarheim, H., Høivik, T., Harthug, S., Digranes, A., Mylvaganam, H., \& Vindenes, H. (2000). [Outbreak of multiresistant Acinetobacter baumannii infection]. Tidsskrift for den Norske laegeforening: Tidsskrift for praktisk medicin. Ny Raekke, 120(9), 1028-1033.

Park, G. C., Choi, J., Jang, S. J., Jeong, S. H., Kim, C. M., Choi, I. S., ... Moon, D. S. (2016). In Vitro Interactions of Antibiotic Combinations of Colistin, Tigecycline, and Doripenem Against Extensively Drug-Resistant and Multidrug-Resistant Acinetobacter baumannii. Annals of laboratory medicine, 36(2), 124-130. http://dx.doi.org/10.3343/alm.2016.36.2.124

Park, Y. K., Jung, S. I., Park, K. H., Cheong, H. S., Peck, K. R., Song, J. H., \& Ko, K. S. (2009). Independent emergence of colistin-resistant Acinetobacter spp. isolates from Korea. Diagnostic microbiology and infectious disease, 64(1), 43-51. http://dx.doi.org/10.1016/j.diagmicrobio.2009.01.012

Perez, F., Hujer, A. M., Hujer, K. M., Decker, B. K., Rather, P. N., \& Bonomo, R. A. (2007). Global challenge of multidrug-resistant Acinetobacter baumannii. Antimicrobial agents and chemotherapy, 51(10), 3471-3484. http://dx.doi.org/10.1128/AAC.01464-06

Peterson, A., Fesik, S., \& McGroarty, E. (1987). Decreased binding of antibiotics to lipopolysaccharides from polymyxin-resistant strains of Escherichia coli and Salmonella typhimurium. Antimicrobial agents and chemotherapy, 31(2), 230-237. http://dx.doi.org/10.1128/AAC.31.2.230

Ramphal, R., \& Ambrose, P. G. (2006). Extended-spectrum $\beta$-lactamases and clinical outcomes: Current data. Clinical infectious diseases, 42(Supplement 4), S164-S172. http://dx.doi.org/10.1086/500663

Seward, R. J., \& Towner, K. J. (1998). Molecular epidemiology of quinolone resistance in Acinetobacter spp. Clinical microbiology and infection, 4(5), 248-254. http://dx.doi.org/10.1111/j.1469-0691.1998.tb00052.x

Shakibaie, M. R., Adeli, S., \& Salehi, M. H. (2012). Antibiotic resistance patterns and extended-spectrum b-lactamase production among Acinetobacter spp. isolated from an intensive care Unit of a hospital in Kerman, Iran. Antimicrobial Resistance Infection Control, 1(1), 1. http://dx.doi.org/10.1186/2047-2994-1-1

Urban, C., Mariano, N., Rahal, J. J., Ponio, C., Koprivnjak, T., \& Weiss, J. (2001). Polymyxin B-Resistant Acinetobacter baumanniiClinical Isolate Susceptible to Recombinant BPI21 and Cecropin P1. Antimicrobial agents and chemotherapy, 45(3), 994-995. http://dx.doi.org/10.1128/AAC.45.3.994-995.2001

Vila, J., Marcos, A., Marco, F., Abdalla, S., Vergara, Y., Reig, R., . . De Anta, T. J. (1993). In vitro antimicrobial production of beta-lactamases, aminoglycoside-modifying enzymes, and chloramphenicol acetyltransferase by and susceptibility of clinical isolates of Acinetobacter baumannii. Antimicrobial agents and chemotherapy, 37(1), 138-141. http://dx.doi.org/10.1128/AAC.37.1.138

Vila, J., Ruiz, J., Go-i, P., \& de Anta, T. J. (1997). Quinolone-resistance mutations in the topoisomerase IV parC gene of Acinetobacter baumannii. Journal of Antimicrobial Chemotherapy, 39(6), 757-762. 
http://dx.doi.org/10.1093/jac/39.6.757

Walsh, T. (2005). The emergence and implications of metallo- $\beta$-lactamases in Gram-negative bacteria. Clinical microbiology and infection, 11(s6), 2-9. http://dx.doi.org/10.1111/j.1469-0691.2005.01264.x

Zheng, W., Yuan, S., \& Li, L. (2013). Analysis of hospital departmental distribution and antibiotic susceptibility of Acinetobacter isolated from sputum samples. American journal of infection control, 41(8), e73-e76. http://dx.doi.org/10.1016/j.ajic.2012.11.004

\section{Copyrights}

Copyright for this article is retained by the author(s), with first publication rights granted to the journal.

This is an open-access article distributed under the terms and conditions of the Creative Commons Attribution license (http://creativecommons.org/licenses/by/3.0/). 